\title{
13. ENTASIS OF A COLUMN
}

Another curve of interest to wood turners and plasterers is the slight curve which can be seen along the length of a column. This curve is called the entasis and is essential in the construction if the column is to appear correct and not have the appearance of being narrower at its centre than at the top end. There are two methods traditionally used to set out the entasis, the first is shown in figure 107(a).

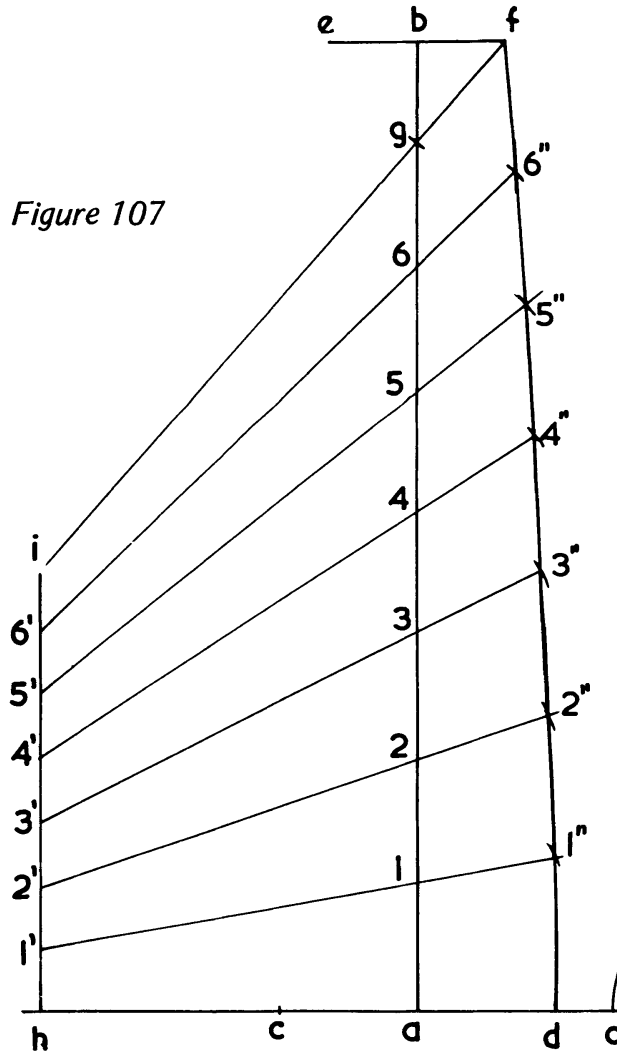

(a)

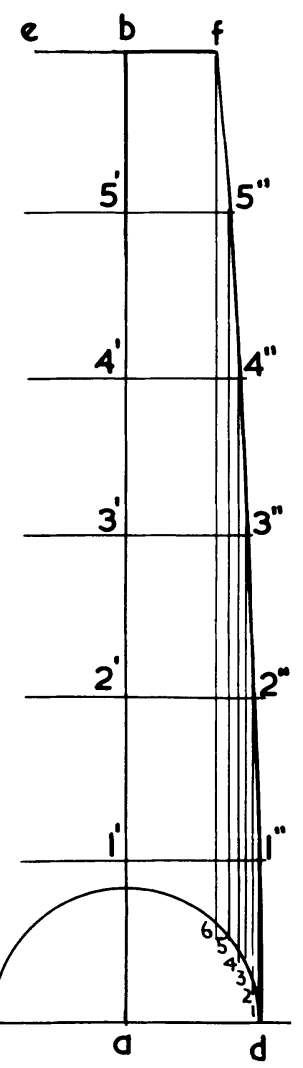

(b)
1. Let $a-b$ be the centre line of the shaft of the column, $c-d$ the diameter at the lower end and $e-f$ the diameter at the top.

2. Divide the centre line $a-b$ into, say, eight equal parts and make $f-g$ equal $a-c$, that is, the radius of the foot of the column.

3. Draw $\mathrm{h}-\mathrm{i}$ parallel to $\mathrm{a}-\mathrm{b}$ some distance away and extend $f-g$ to give $i$ on $h-i$.

4. Divide $\mathrm{h}-\mathrm{i}$ into the same number of equal parts as $a-b$ and join 6 to $6^{\prime}$ etc., extending the lines as shown.

5. With radius $f-g$ and centres $1,2,3 \ldots$ in turn, describe the arcs to cut the extended lines at points $1^{\prime \prime}, 2^{\prime \prime}, 3^{\prime \prime}$ etc. Join these points to produce the entasis.

The second method can be seen in figure 107(b):

1. Draw the centre line of the column $a-b$ and divide this into, say, six equal parts. Draw horizontal lines through these points.

2. Make $c-d$ the diameter at the lower end and $e-f$ the diameter at the top.

3. Draw the semi-circle $c-d$ and drop a vertical line from $f$ to give 6 on the semi-circle.

4. Divide $d-6$ into the same number of parts as was the centre line $a-b$, in this case, six.

5. Project vertical lines from the points on the semi-circle upwards to the horizontal lines along the length of the column. 1 and 1' will meet at 1", 2 and 2' will give 2", etc. These points will give the outline of the entasis. 